\title{
Nanostructured Zinc Oxide Synthesized via Hydroxide Route as Liquid Petroleum Gas Sensor
}

\author{
Bal Chandra Yadav*, Richa Srivastava and Anuradha Yadav \\ Nanomaterials and Sensors Research Laboratory \\ Department of Physics, University of Lucknow, Lucknow-226007, U.P., India
}

(Received April 12, 2008; accepted November 10, 2008)

Key words: $\quad$ LPG sensor, ZnO nanomaterial, SEM, XRD

In this paper, we report the liquid petroleum gas (LPG) sensing of nanostructured zinc oxide synthesized via the hydroxide route. Variations in resistance with exposure of gas to the sensing element have been observed. The average sensitivities for different exposure times of 2,5 , and $8 \mathrm{~min}$ to gas have been estimated. The maximum average sensitivity observed is $7.81 \mathrm{M} \Omega / \mathrm{min}$ for an exposure time of $8 \mathrm{~min}$ to the sensor. Sensor response $(S R)$ as a function of exposure time to the gas has been calculated. Scanning electron microscopy (SEM) and X-ray diffraction studies of samples have been carried out. SEM images show that the material is porous and has a nanosheet-like morphology before exposure to the LPG. The thicknesses of the nanosheets vary from 60-200 $\mathrm{nm}$. Nanowires of different diameters of $\mathrm{ZnO}$ can be observed after exposure to LPG. The diameters of these nanowires vary from $80-300 \mathrm{~nm}$. XRD patterns reveal the amorphous nature of the material. The sensor is quite sensitive to LPG, and the results are reproducible. Furthermore, the LPG sensor reported is cost-effective, user friendly, and easy to fabricate.

\section{Introduction}

Nanosized metal oxides have been used to produce low-cost gas sensing materials. ${ }^{(1)}$ Metal oxide semiconductors as gas sensing materials have been extensively studied for a long time because of their important features such as stability, good sensitivity to ambient conditions, and simplicity of fabrication. ${ }^{(1-3)}$ Several investigations have been carried out to understand and improve the gas sensing properties, particularly for inflammable and toxic gas detection, but only a few published reports are available on designing a sensor for the detection of liquid petroleum gas (LPG).(2) Semiconductor gas sensors based on the controlled electrical conductivity upon exposure to gases have attracted considerable attention because of their compact size, which facilitates the miniaturization of electronic circuits and simplifies the sensing method. Nanomaterials have been developed for gas sensing in which metal oxides that are physically and chemically stable have been

*Corresponding author: e-mail: balchandra_yadav@rediffmail.com 
investigated extensively.(4,5)

Metal oxide semiconductors are in vogue for the detection of combustible gases by a change in the surface conductivity due to exposure to gas. $\mathrm{ZnO}, \mathrm{SnO}_{2}, \mathrm{TiO}_{2}, \mathrm{CuO}$, and $\mathrm{Fe}_{2} \mathrm{O}_{3}$ have been investigated as sensors for water vapour, $\mathrm{O}_{2}, \mathrm{H}_{2}, \mathrm{NO}_{x}, \mathrm{EtOH}$, and LPG. ${ }^{(5-8)}$ One of the requirements of a gas sensor is low power consumption because the sensor needs to work reliably and continuously. Among these materials, $\mathrm{ZnO}$ has promise to fulfill these requirements. $\mathrm{ZnO}$ displays a variety of morphologies and a large range of promising device applications. It is an n-type material of wurtzite structure. It has a direct band gap of $3.37 \mathrm{eV}$ at room temperature and a relatively high excitation energy $(60 \mathrm{MeV})$. ZnO-based elements have attracted much attention as gas sensors because of their chemical sensitivity to volatile gases, their high chemical stability, suitability for doping, lack of toxicity, and low cost. ${ }^{(9-12)}$ Since we know that an ideal gas sensor should have the ability to discriminate between various gases, with this in mind, $\mathrm{ZnO}$ behaves like a good sensing material. ${ }^{(4,5,13-20)}$

\section{Materials and Methods}

\subsection{Synthesis}

$\mathrm{ZnO}$ is prepared by a conventional precipitation method via the hydroxide route. ${ }^{(21)} \mathrm{A}$ pellet of $\mathrm{ZnO}$ powder with $10 \%$ glass powder as binder has been made using a hydraulic pressure machine (MB Instruments, India) at a pressure of $30 \mathrm{MPa}$ at room temperature. The addition of a glass binder during the process plays an important role in promoting adhesion of the material for pellet formation.

\subsection{Characterization}

Figure 1 shows a SEM image of the sensing material before exposure to LPG. A nanosheet-like morphology of $\mathrm{ZnO}$ is observed. The thicknesses of the nanosheets vary from 60 to $200 \mathrm{~nm}$. Figures 2(a)-2(c) show the SEM images of the sensing material after exposure to the LPG at different scales and magnifications. After exposure to LPG, the nanosheet-like

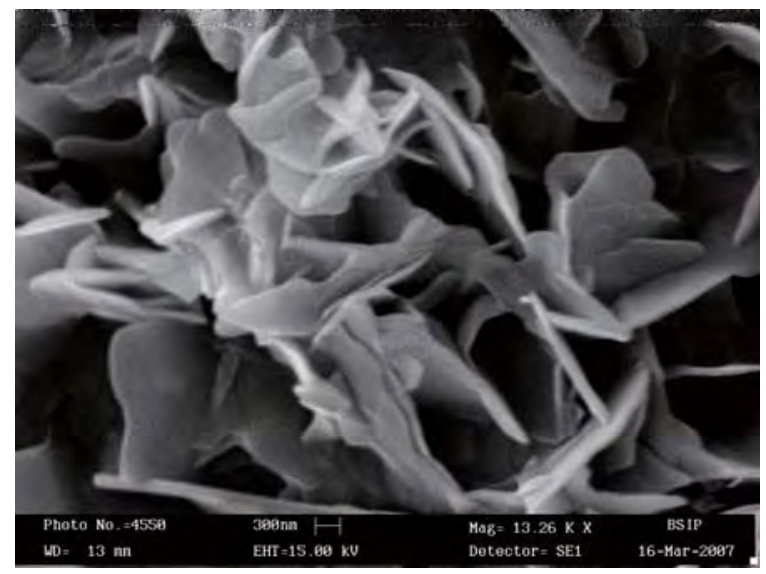

Fig. 1. SEM image of $\mathrm{ZnO}$ before exposure to $\mathrm{LPG}$ at room temperature. 
(a)

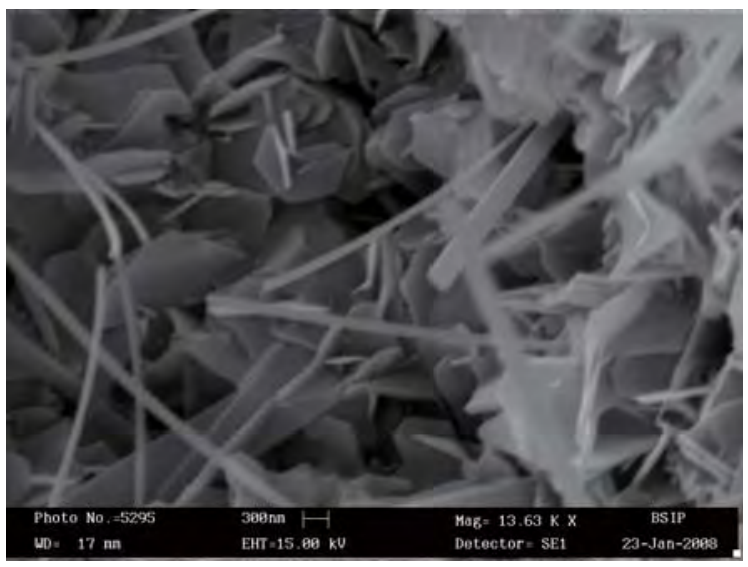

(b)

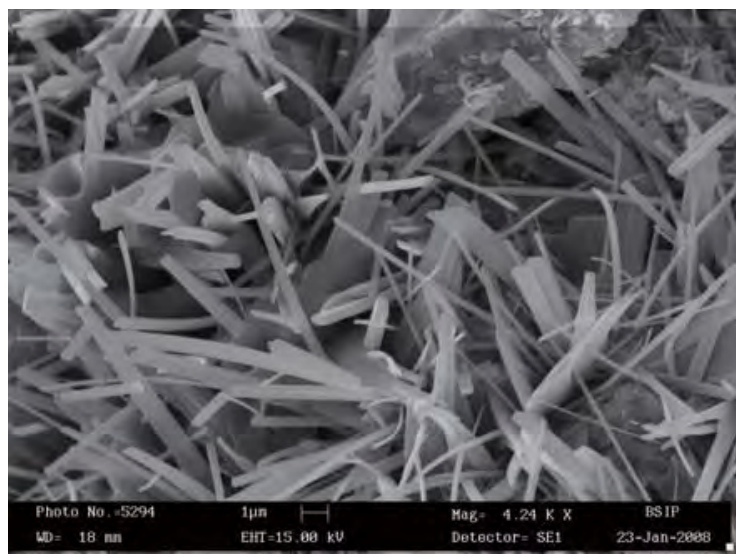

(c)

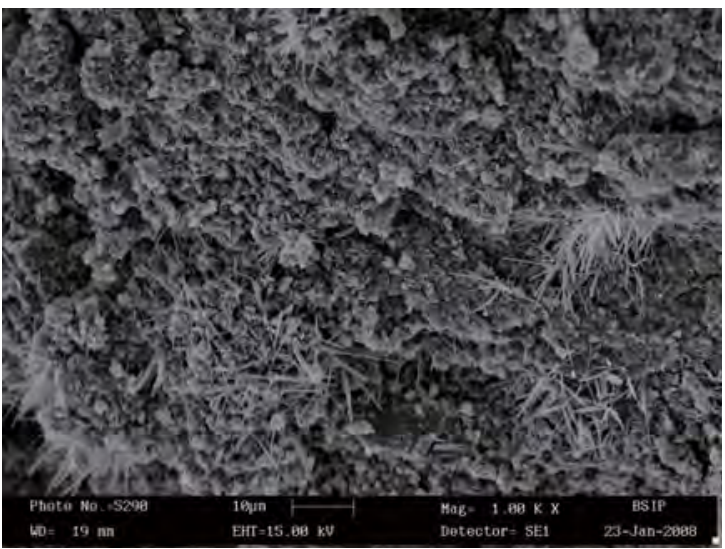

Fig. 2. SEM images of $\mathrm{ZnO}$ after exposure to LPG: (a) nanoscale, (b) $1 \mu \mathrm{m}$ scale, and (c) $10 \mu \mathrm{m}$ scale. 
structures of $\mathrm{ZnO}$ change into nanowires having diameters from 70 to $300 \mathrm{~nm}$.

Figure 3 shows the X-ray diffraction pattern of the sensing material. This image reveals the amorphous nature of the material, and a major part of the material consists of the $\mathrm{ZnO}$ nanophase. The major peaks identified are at $2 \theta=32^{\circ}$ with ' $\mathrm{d}$ ' spacing and full width at half maximum (FWHM) of $2.82 \AA$ and $0.283^{\circ}$, respectively, corresponding to plane (100), and at $2 \theta=34^{\circ}$ with ' $d$ ' spacing and FWHM $2.61 \AA$ and $0.283^{\circ}$, respectively, corresponding to plane (002). The other intense peak is at $2 \theta=36^{\circ}$ with ' $\mathrm{d}$ ' spacing and FWHM $2.47 \AA$ and $0.315^{\circ}$, respectively, corresponding to plane (101).

\subsection{Experimental method}

The schematic diagram of the experimental setup is shown in Fig. 4. The heart part of the device is the conductivity-measuring pellet holder. It is fitted well in a glass

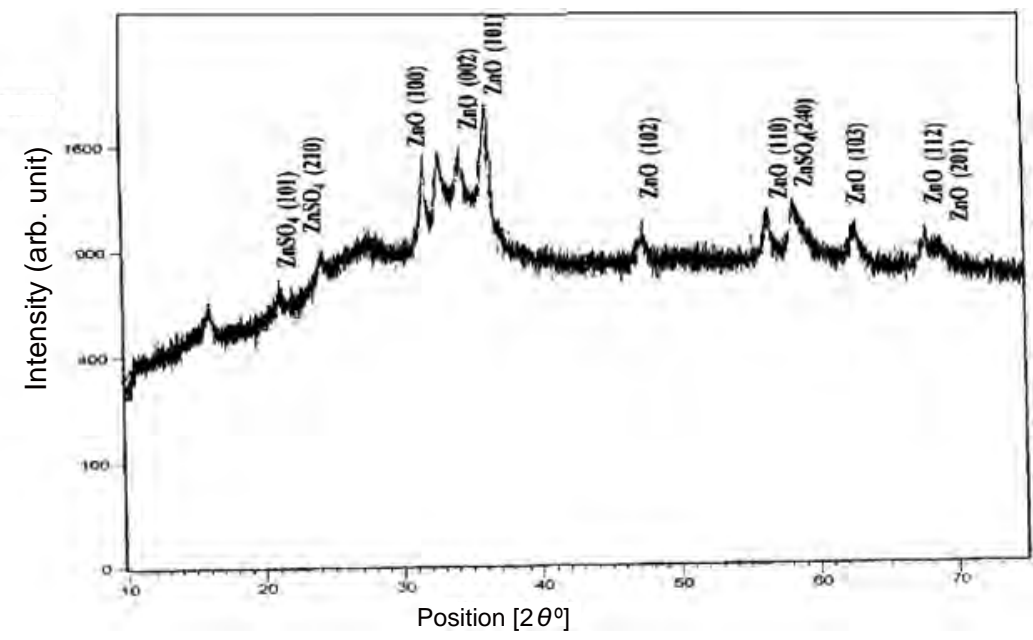

Fig. 3. X-ray diffraction pattern of sensing material at room temperature.

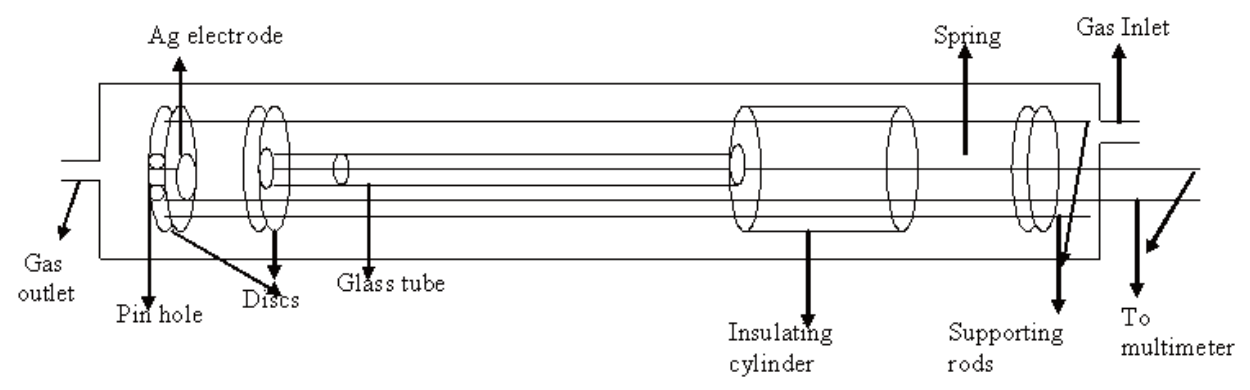

Fig. 4. Device assembly for gas sensing. 
chamber having inlet and outlet knobs for LPG. The inlet knob is associated with a concentration measuring system along with a thermocouple. The pellet was placed in the Ag-pellet-Ag electrode configuration. It was exposed to LPG in a specially designed chamber under controlled conditions, and the corresponding variation in resistance with the exposure time to LPG was recorded using a digital multimeter (VC 9808, India). The sensitivity $(S)$ is defined as the ratio of change in the resistance to the exposure time;

$$
S=\Delta R / \Delta t \quad(\mathrm{M} \Omega / \mathrm{min})
$$

The sensor response of a sensing element is defined as

$$
S R=\left|R_{\mathrm{a}}-R_{\mathrm{g}}\right| / R_{\mathrm{a}},
$$

where $R_{\mathrm{a}}$ is the resistance of the sensing element in air and $R_{\mathrm{g}}$ is the resistance in gas.

\section{Results and Discussion}

The resistance of the sensing material increases with time of exposure to the gas. The variation in resistance with time of exposure to the gas through the sensing element has been observed as shown in Fig. 5. In general, as the exposure time increases, the resistance of the sensing element increases. For an exposure time of 2 min shown in curve 'a,' there is a regular increase in resistance with a slow sensor response. Curve ' $b$ ' shows a similar behavior with a slightly improved sensor response, and curve ' $c$ '

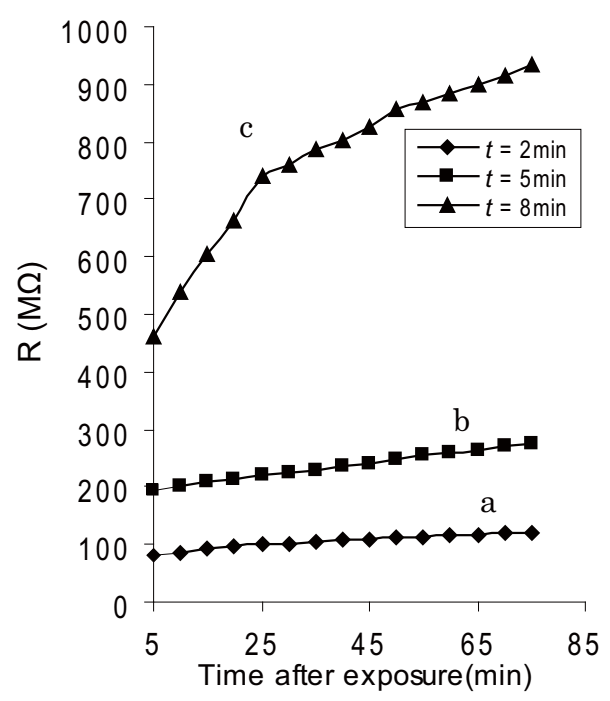

Fig. 5. Variations in resistance of $\mathrm{ZnO}$ sensing element with exposure time. 
exhibits a marked increase in resistance with an exposure time of $8 \mathrm{~min}$. The variation in average sensitivity with different exposure times is shown in Fig. 6. The highest average sensitivity of $7.81 \mathrm{M} \Omega / \mathrm{min}$ of a sensor was observed for an exposure time of $8 \mathrm{~min}$. The sensor response properties were studied. The sensor response curve for different exposure times is also shown in Fig. 7. From the curve, it is clear that this sensing element also has a maximum sensing response for an exposure time of $8 \mathrm{~min}$.

The working principle of the semiconducting gas sensors is based on the change in the electronic conductivity of the semiconducting material upon exposure to the gas. The interaction of gas molecules with the surface of the pellet causes the transfer of electrons between the semiconducting surface and the reducing gases. Atmospheric oxygen molecules $\left(\mathrm{O}_{2}\right)$ are adsorbed by the surface of the pellet of $\mathrm{ZnO}$. They capture the electrons from the conduction band of the sensing material as

$$
\mathrm{O}_{2 \text { (air) }}+4 e^{-} \rightarrow 2 \mathrm{O}^{2-} \text { (pellet surface) }
$$

Because of this, the electronic conductivity decreases, which increases the resistance of the sensing material. When LPG reacts with the oxygen of $\mathrm{ZnO}$, a complex series of reactions take place. The reaction of LPG molecules with adsorbed oxygen is

$$
\mathrm{C}_{n} \mathrm{H}_{2 n+2}+2 \mathrm{O}^{2-} \leftrightarrow \mathrm{H}_{2} \mathrm{O}+\mathrm{C}_{n} \mathrm{H}_{2 n}-\mathrm{O}+e^{-}
$$

Here, $\mathrm{C}_{n} \mathrm{H}_{2 n+2}$ represents various hydrocarbons. ${ }^{(22)}$

Thus, a single molecule of LPG liberates $1 e^{-}$in the conduction band of $\mathrm{ZnO}$. As the pressure of the gas inside the chamber increases, the rate of adsorption increases

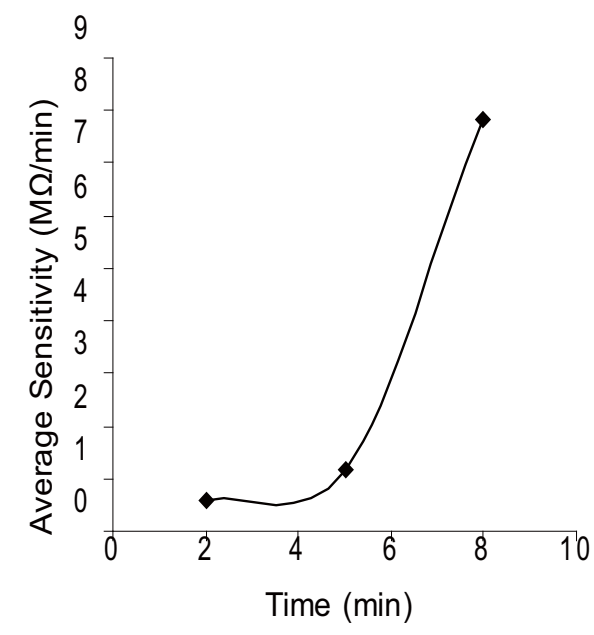

Fig. 6. Variation of average sensitivity with different exposure times to LPG. 


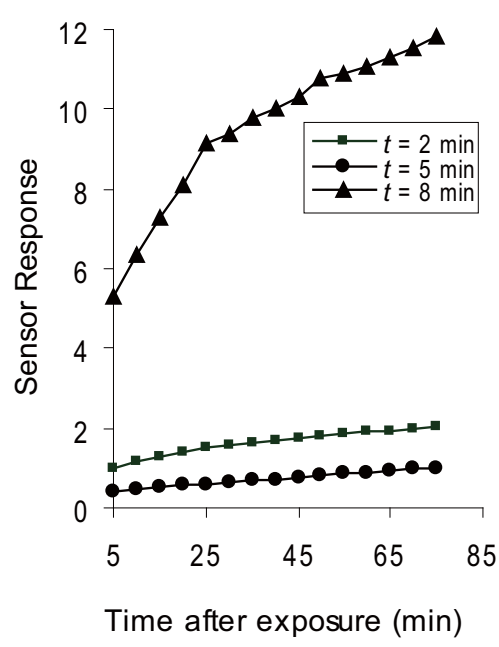

Fig. 7. Sensor response curves for different exposure times to LPG.

resulting in the withdrawal of electrons from the conduction band of the $\mathrm{ZnO}$ and the development of a potential barrier to charge transport. Therefore, the resistance of the sensing material increases with exposure to the gas.

\section{Conclusions}

The LPG sensor reported here shows the highest sensitivity of $7.81 \mathrm{M} \Omega / \mathrm{min}$ for the maximum exposure time. Although the observations are in terms of exposure time and not in terms of gas concentration, it is still very useful for the detection of liquid petroleum gas. The leakage time is very important for disaster management purposes, and that is the reason this study has potential commercial applications.

\section{References}

1 Y. Li, G. S. Tompa, S. Liang, C. Gorla, Y. Lu and J. Doyle: J. Vac. Sci. Technol., A 15 (1997) 1063.

2 P. Bhattacharya, P. K. Basu, C. Lang, H. Saha and S. Basu: Sens. Actuators, B 129 (2008) 551.

3 N. Barsan, D. Koziej and U. Weimar: Sens. Actuators, B 121 (2007) 18.

4 B. Baruwati, D. K. Kumar and S.V. Manorama: Sens. Actuators, B 119 (2006) 676.

5 V. R. Shinde, T. P. Gujar and C. D. Lokhande: Sens. Actuators, B 120 (2007) 551.

6 I. Stambolova, K. Konstantinov, S. Vassilev, P. Peshev and T. Tsacheva: Mater. Chem. Phy. 63 (2000) 104.

7 H. Nanto, T. Minami and S. Tokata: J. Appl. Phys. 60 (1986) 482 
8 B. C. Yadav, R. Srivastava, C. D. Dwivedi and P. Pramanik: Sens. Actuators B: Chem. 130 (2008) 216.

9 T. Seiyama, A. Kato, K. M. Fujiishi and M. Nagatani: Anal. Chem. 34 (1962) 1502.

10 N. Yamazoe, G. Sakai and K. Shimanoe: Catal. Surveys Asia 1 (2003) 63.

11 P. Zu, Z.K. Tang, G. K. L. Wong, M. Kawasaki, A. Ohtomo and K. Koinumal.: Solid State Commun. 103 (1997) 459.

12 D. M. Bagnall, Y. R. Chen, Z. Zhu, T. Yao, S. Koyama and M. Y. Shen.: Appl. Phys. Lett. 70 (1997) 2230.

13 V. R. Shinde, T. P. Gujar and C.D. Lokhande: Sens. Actuators, B 123 (2007) 882.

14 B. Li-Jian, Y. Xiao-Na, D. Yue-Qin and Y. Zhi-Hao: Sens. Actuators, B 126 (2007) 604.

15 D. R. Patil, L. A. Patil, G. H. Jain, M. S. Wagh and S. A. Patil: Sens. Trans. J. 74 (2006) 874.

16 D. R. Patil, L. A. Patil and P. P. Patil: Sens. Actuators, B 126 (2007) 368.

17 D. R. Patil and L. A. Patil: Sens. Actuators, B 123 (2007) 546.

18 S. Kucheyev, J. E. Bradley, J. S. Williams, C. Jagdish and M. V. Swain: Appl. Phys. Lett. 80 (2002) 956.

19 M. Wraback, H. Shen, S. Liang, C. R. Gorla and Y. Lu: Appl. Phys. Lett. 76 (2000) 3257.

20 P. M. Verghese and D. R. Clarke: J. Appl. Phys. 87 (2000) 4430.

21 B. C. Yadav, R. Srivastava and C. D. Dwivedi: Syn. and React. in Inorg. Met.-Org. and NanoMetal Chem. 37 (2007) 1.

22 V. R. Shinde, T. P. Gujar and C. D. Lokhande: Sens. Actuators, B 2 (2006) 701. 